PREPARED FOR THE U.S. DEPARTMENT OF ENERGY, UNDER CONTRACT DE-AC02-76CH03073

PPPL-3846

PPPL-3846

UC-70

Current Drive in a Ponderomotive Potential with Sign Reversal

by

N.J. Fisch, J.M. Rax, and I.Y. Dodin

July 2003

N/M

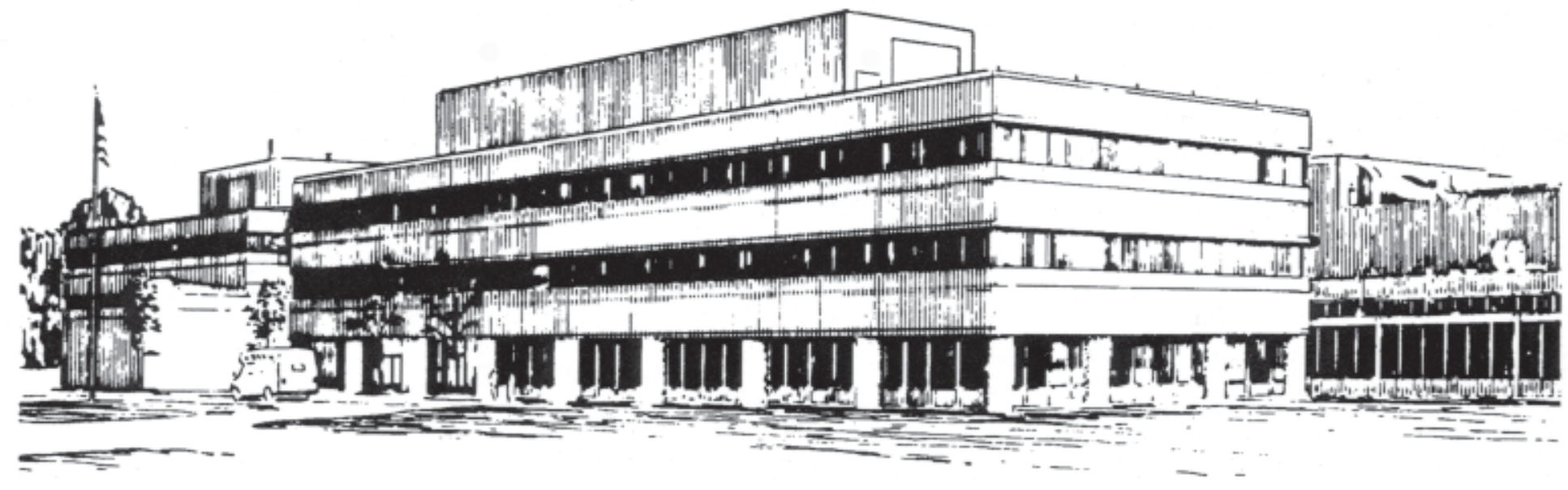

PRINCETON PLASMA PHYSICS LABORATORY PRINCETON UNIVERSITY, PRINCETON, NEW JERSEY 


\section{PPPL Reports Disclaimer}

This report was prepared as an account of work sponsored by an agency of the United States Government. Neither the United States Government nor any agency thereof, nor any of their employees, makes any warranty, express or implied, or assumes any legal liability or responsibility for the accuracy, completeness, or usefulness of any information, apparatus, product, or process disclosed, or represents that its use would not infringe privately owned rights. Reference herein to any specific commercial product, process, or service by trade name, trademark, manufacturer, or otherwise, does not necessarily constitute or imply its endorsement, recommendation, or favoring by the United States Government or any agency thereof. The views and opinions of authors expressed herein do not necessarily state or reflect those of the United States Government or any agency thereof.

\section{Availability}

This report is posted on the U.S. Department of Energy's Princeton Plasma Physics Laboratory Publications and Reports web site in Fiscal Year 2003. The home page for PPPL Reports and Publications is: http://www.pppl.gov/pub_report/

DOE and DOE Contractors can obtain copies of this report from:

U.S. Department of Energy

Office of Scientific and Technical Information

DOE Technical Information Services (DTIS)

P.O. Box 62

Oak Ridge, TN 37831

Telephone: (865) 576-8401

Fax: (865) 576-5728

Email: reports@adonis.osti.gov

This report is available to the general public from:

National Technical Information Service

U.S. Department of Commerce

5285 Port Royal Road

Springfield, VA 22161

Telephone: $1-800-553-6847$ or

(703) $605-6000$

Fax: (703) 321-8547

Internet: http://www.ntis.gov/ordering.htm 


\title{
Current Drive in a Ponderomotive Potential with Sign Reversal
}

\author{
N. J. Fisch ${ }^{(a)}$, J. M. Rax ${ }^{(b)}$, and I. Y. $\operatorname{Dodin}^{(a)}$ \\ ${ }^{(a)}$ Princeton Plasma Physics Laboratory, Princeton University, Princeton, NJ 08543 \\ ${ }^{(b)}$ LPTP, Ecole Polytechnique, 91128 Palaiseau, France
}

(Dated: May 7, 2003)

\begin{abstract}
Noninductive current drive can be accomplished through ponderomotive forces with high efficiency when the potential changes sign over the interaction region. The effect can practiced upon both ions and electrons. The current drive efficiencies, in principle, might be higher than those possible with conventional rf current drive techniques, since different considerations come into play.
\end{abstract}

PACS numbers: 52.55.Wq, 52.35.Mw, 52.40.Db

Radio frequency (rf) waves can drive currents efficiently in plasma in several frequency regimes [1-3]. The most promising effects rely upon resonant wave-particle interactions: In the range of the lower hybrid frequency, electrostatic waves with high phase velocity can efficiently drive superthermal electrons in the direction of the wave phase velocity [1]. In the range of the electron cyclotron frequency, the current drive effect relies upon the perpendicular heating of electrons traveling in one toroidal direction [2]. In the ion cyclotron frequency regime, low frequency waves can resonantly drive either electrons [4] or ions [5] to produce current drive effects. All of these effects enjoy considerable experimental verification.

The efficiency in any resonant $\mathrm{rf}$ current drive is limited in that it is essentially a diffusive process, with low energy particles propelled to higher energy to drive current. In the absence of a population inversion, kinetic energy to the particles must be provided by the wave. (The exception to this rule is when a population inversion exists along a diffusion path that connects to a low density region [6].) These current drive schemes tend to be less efficient than is current drive with a dc electric field, since a dc electric field drives all electrons in the same direction. Hence, in the case of a dc field, momentum is imparted by braking electrons traveling against the force field, thereby extracting kinetic energy rather than by providing kinetic energy to half of the electrons.

The question is whether higher efficiencies can be achieved via the so-called "ponderomotive" forces, which have been studied in a number of contexts [7-31]. These possibilities include the use of the non-resonant ponderomotive forces in driving plasma current [7-11]. Litwin [12] suggests that there is cancellation that reduces the so-called alpha effect relied upon by others [9]. What we show, however, is that the current drive effect can be achieved efficiently in an inhomogeneous magnetic field, where an important asymmetry of the ponderomotive potential may be realized [7], rather than in the absence of a magnetic field or in the presence of only a uniform magnetic field, where this effect cannot be realized.

To show the current drive effect in an inhomogeneous field, suppose a plasma is immersed in a magnetic field $\boldsymbol{B}_{0}$ largely in the $z$-direction, with some variation in $z$, so that $\boldsymbol{B}_{0} \approx$ $B_{0}(z) \hat{z}$. The cyclotron frequency of a particle with charge $e$ and mass $m$ is then $\Omega(z)=e B_{0}(z) / m c$. Suppose an elec- tric field spectrum of the form $\boldsymbol{E}_{\mathrm{rf}}=\hat{\boldsymbol{x}} E_{x}(z) \sin \omega t$ and the consistent magnetic field given by $\partial \boldsymbol{B}_{\mathrm{rf}} / \partial t=-c \nabla \times \boldsymbol{E}_{\mathrm{rf}}$. This imposed field is assumed for simplicity in demonstrating the current-drive effect; a more precise calculation, beyond the scope of this effort, should consider the propagation of realizable fields, obeying the plasma dispersion relation. In the magnetic field and electric field imposed here, the charged particle experiences an average (over the rf period) "ponderomotive" potential, which may be written as

$$
\Phi(z)=\frac{1}{4} m v_{\mathrm{osc}}^{2}(z) \frac{\omega^{2}}{\omega^{2}-\Omega^{2}(z)},
$$

where $v_{\text {osc }}^{2} \equiv\left(e E_{x} / m \omega\right)^{2}$. A charged particle, in the ponderomotive potential (1), exhibits reversible motion if the beat frequency $\omega-\Omega$ changes little in a period, i.e., $v_{z} \Omega^{\prime}(z) /(\omega-$ $\Omega)^{2} \ll 1$, where $v_{z}$ is the particle velocity along the magnetic field [24]. Near the cyclotron resonance, $\Omega=\omega$, this condition is clearly violated, resulting in chaotic motion of the particle. The characteristic width of the resonant interaction region is given by $\delta z=\sqrt{L_{B} v_{z} / \omega}$, where $L_{B}$ is the characteristic scale of the dc magnetic field.

Note that the potential given by Eq. (1) is asymmetric in the $z$-direction, as shown in Fig. 2. Ponderomotive potentials of this form have been proposed for rf confinement of plasma (for a review, see Ref. [7]) and stabilization of lowfrequency modes in magnetically confined plasmas [23], as well as for isotope separation in plasmas composed of multiple ion species [18]. These applications make use of the enhancement of the potential near the resonance, rather than the sign reversal. What we show here is that it is precisely the sign-reversal in the potential that can be exploited to produce a non-resonant current drive effect with high efficiency.

Assume a $z$-dependence of electric field $E_{x}(z)$ and magnetic field $B_{0}(z)$ such that, at $z=0, E_{x}(z)$ has a maximum and $\omega=\Omega$ (see Fig. 1). Define the region I (say $z<z_{1}$ ), for which $\omega<\Omega(z)$, and for which the particle motion is essentially adiabatic. Similarly, define the adiabatic region III (say $z>z_{2}$ ), for which $\omega>\Omega(z)$. Define resonant region II, $z_{1}<z<z_{2}$, for which the particle is essentially in resonance $\left(z_{2}-z_{1} \sim \delta z\right)$. Particles encountering the adiabatic regions may be reflected, whereas upon traversing the resonant region, they are subject to perpendicular heating. Electrons impinging on the ponderomotive region traveling in the $-\hat{\boldsymbol{z}}$ direction 


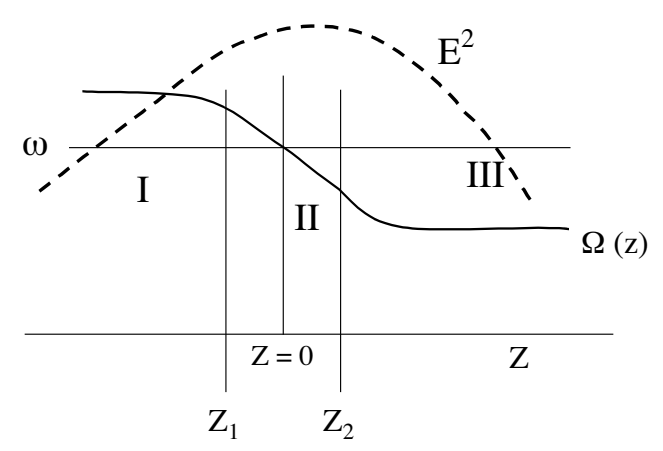

FIG. 1: Schematic of the magnetic field configuration. The electric field energy profile is shown by dotted lines. The maximum of the electric field occurs at $z=0$, which is also where the local gyrofrequency coincides with the frequency of the applied electric field.

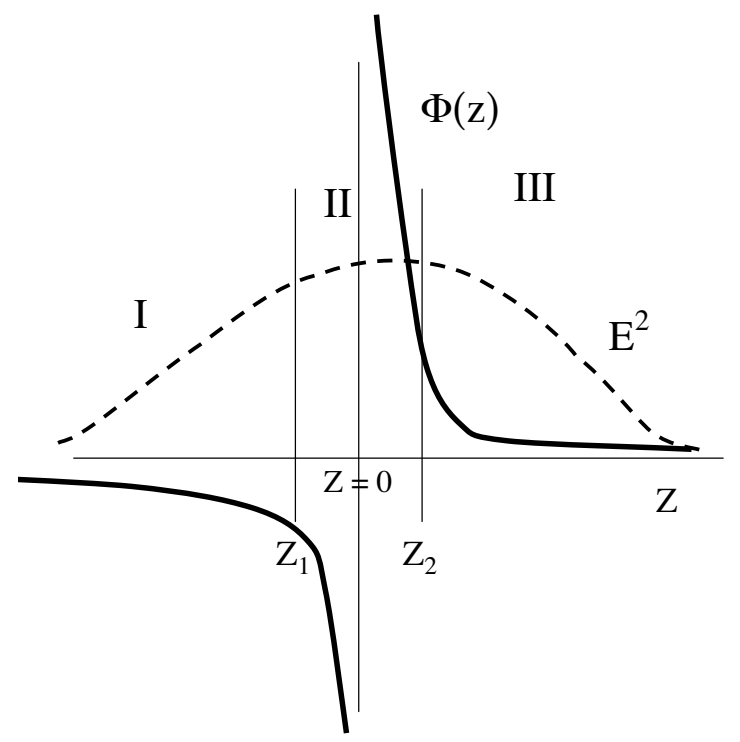

FIG. 2: Schematic of the ponderomotive potential $\Phi(z)$ (solid line) for the electric and magnetic fields given in Fig. 1.

can be reflected, but because of the sign reversal, electrons encountering the barrier traveling in the $+\hat{\boldsymbol{z}}$ direction will be further accelerated precisely in the $+\hat{\boldsymbol{z}}$ direction. Thus, such a barrier, operating essentially as a Maxwell's demon, might be very efficient in generating current.

In addition to the ponderomotive force, a magnetic force $\boldsymbol{F}=-\mu \nabla B_{0}$ accelerates particles along the dc magnetic field, where the quantity $\mu=m u_{\perp}^{2} / 2 B_{0}$ is an approximate integral of particle motion [7], analogous to the adiabatic invariant of free gyromotion in a slowly varying magnetic field. (Here $\boldsymbol{u}_{\perp}=\boldsymbol{v}_{\perp}-\boldsymbol{v}_{\mathrm{rf} \perp}$ is the perpendicular velocity in the absence of the velocity oscillation, $\boldsymbol{v}_{\mathrm{rf}}$, due to the rf field.) Note from Fig. 2 that both the magnetic $\left(\mu \nabla B_{0}\right)$ and ponderomotive forces $(\nabla \Phi)$ tend to drive particles in the $+\hat{\boldsymbol{z}}$ direction in the regions, where the ponderomotive potential is established (regions I and III). Inside the region II, where the ponderomotive approximation does not hold, the force ex-

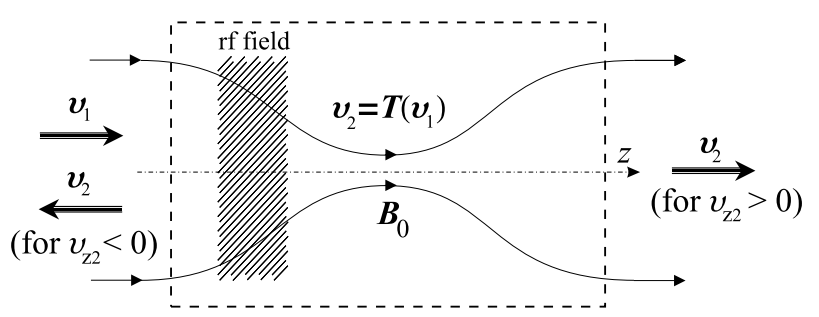

FIG. 3: Region of current drive excitation. The magnetic field going in and out of the excitation region is of the same magnitude and direction. The field lines return on themselves outside of the current drive region. Particle can be either reflected or transmitted by the rf barrier. The particle velocity $\boldsymbol{v}_{2}$ after scattering is mapped by the nonlinear operator $\boldsymbol{T}$ to the velocity $\boldsymbol{v}_{1}$ before scattering.

erted on a particle also has the same sign, since it is primarily the magnetic force, with the magnetic moment $\mu$ changing in time because of resonant interaction with the rf field. Because of these nonadiabatic effects, the integrated magnetic force, $\boldsymbol{F}=-\mu \nabla B_{0}$, does not vanish over a return of the particle to the same magnitude magnetic field.

To calculate the current drive effect produced by the rf barrier, imagine a region in space where the ponderomotive force is applied (see Fig. 3). Suppose that the magnetic field going in and out of this region is of the same magnitude and direction, although the field lines pinch inside the region. In the pinch region, the cyclotron resonance is satisfied locally. Suppose also that collisions are negligible over the time a particle crosses the pinch region. Then, electrons (or ions) coming into this region are either reflected or transmitted, but in any event, the slowing down of the electrons occurs independently of the ponderomotive field. As such, what matters is only that, as a result of encountering the barrier, electrons go from velocity space location $\boldsymbol{v}_{1}$ to location $\boldsymbol{v}_{2}$. If one knew the transformation function $\boldsymbol{v}_{2}=\boldsymbol{T}\left(\boldsymbol{v}_{1}\right)$, one would be able to calculate the efficiency by simply averaging over all particles.

To calculate the current drive efficiency, note that the region of current drive excitation (Fig. 3) can be thought of as a current source. The source operates as a selective barrier. The number of particles in the velocity interval $d^{3} v_{1}$ scattered off the barrier during time $d t$ within the cross-section $d A$ is $d N_{B}=f\left(\boldsymbol{v}_{1}\right)\left|v_{z 1}\right| d^{3} v_{1} d t d A$, where $f\left(\boldsymbol{v}_{1}\right)$ is the electron velocity distribution function.

Suppose the plasma is toroidal with period $2 \pi R$. The instantaneous particle velocity $\bar{v}_{z}$ in the $\hat{z}$ direction changes as a result of collisions with background particles, so that it is a function of both initial coordinates and time, i.e., $\bar{v}_{z}=\bar{v}_{z}(\boldsymbol{v}, t)$, where $\bar{v}_{z}(\boldsymbol{v}, t=0)=v_{z}$. The current carried by $d N_{B}$ electrons beginning with $v_{z}=v_{z 1}$ is then $d I_{z}^{(1)}=e d N_{B} \bar{v}_{z}\left(\boldsymbol{v}_{1}, t\right) / 2 \pi R$. Then the current generated by $d N_{B}$ particles scattering from velocity space location (1) to velocity space location (2) is then given by the difference between the current generated over time by an electron beginning in location (2) and the current generated by an electron beginning in location (1). Under continuous scattering by a 
barrier from location (1) to location (2), the generated current can then be written as

$$
J=\frac{e}{2 \pi R} \int f\left(\boldsymbol{v}_{1}\right)\left[\chi\left(\boldsymbol{v}_{2}\right)-\chi\left(\boldsymbol{v}_{1}\right)\right]\left|v_{z 1}\right| d^{3} v_{1}
$$

where $\chi(v)$ is the current response function:

$$
\chi(\boldsymbol{v})=\int_{0}^{\infty} \bar{v}_{z}(\boldsymbol{v}, t) d t .
$$

For example, for constant slowing down rate $\nu$, we get $\chi\left(v_{z}, v_{\perp}\right)=v_{z} / \nu(\boldsymbol{v})$. For superthermal velocities, the velocity dependence of the collisions cannot be ignored; in the high-velocity limit $\chi\left(v_{z}, v_{\perp}\right) \rightarrow\left(v_{z} / \nu_{0}\right)\left(v / v_{\mathrm{th}}\right)^{3} /\left(5+Z_{i}\right)$, where $Z_{i}$ is the ion charge state, $v_{\text {th }}$ is the electron thermal speed, and $\nu_{0}$ is the collision frequency of thermal electrons [2].

The efficiency of a current drive scheme is determined by how much current can be produced per unit power taken from an rf source. Perfect adiabatic reflection would generate current with no power dissipation, acting like a Maxwell demon, and thus violating thermodynamic laws. However, irreversible heating inevitably accompanies the current drive. There are two sources of heating to consider: One is the heating of the antenna, or whatever is holding the electromagnetic barrier in place, as particles bounce off of it. The other is the stochastic heating of passing particles that are transmitted through the barrier but stochastically heated, as they traverse the resonance. We neglect here the antenna heating, which would only become important in the limit of efficiencies much higher than those generally achievable in driving currents by rf waves. Then, the power dissipated by the rf barrier per unit crosssection is given by

$$
P=\int f\left(\boldsymbol{v}_{1}\right) \Delta \mathcal{E}\left(\boldsymbol{v}_{1}\right)\left|v_{z 1}\right| d^{3} v_{1},
$$

where $\Delta \mathcal{E}\left(\boldsymbol{v}_{1}\right)=m\left(v_{2}^{2}-v_{1}^{2}\right) / 2$ is the average irreversible energy gain of an individual particle as it scatters off the rf barrier.

The current drive efficiency $\eta=J / P$ can be determined precisely for a Maxwellian distribution of electrons in a straightforward if tedious calculation. However, one can give a very rough estimate of the efficiency by considering a model velocity distribution

$$
f(\boldsymbol{v})=n \frac{\delta\left(v_{\perp}-v_{\perp c}\right)}{4 \pi v_{\perp c}}\left[\delta\left(v_{z}-v_{z c}\right)+\delta\left(v_{z}+v_{z c}\right)\right]
$$

where the positive quantities $v_{\perp c}$ and $v_{z c}$ stand for the characteristic transverse and longitudinal electron velocities of the order of $v_{\text {th }}$. Substituting (5) into Eqs. (2), (4) and using $\chi\left(v_{\perp},-v_{z}\right)=-\chi\left(v_{\perp}, v_{z}\right)$ one gets

$$
\eta=\frac{e}{2 \pi R} \frac{\chi\left(\boldsymbol{v}_{+}\right)+\chi\left(\boldsymbol{v}_{-}\right)}{\Delta \mathcal{E}_{+}+\Delta \mathcal{E}_{-}}
$$

where $\boldsymbol{v}_{ \pm} \equiv \boldsymbol{T}\left(v_{\perp c}, \pm v_{z c}\right)$, and $\Delta \mathcal{E}_{ \pm}$stand for the energy change of a particle with initial velocity $\left(v_{\perp c}, \pm v_{z c}\right)$. Note that, since we derived this formula without specifying the actual mapping $\boldsymbol{T}(\boldsymbol{v})$, Eq. (6) is applicable for estimating the current drive efficiency for a variety of mechanisms causing the heating and acceleration of charged particles, as long as those processes can be described by a velocity space mapping.

How large is the efficiency of the current drive in a ponderomotive potential with sign reversal? The largest efficiency is achieved in the regime where the heating is brought to minimum. Suppose that the effective height of the ponderomotive potential $\Phi_{\max }[7,26]$ is larger than the electron thermal energy: $\kappa=\Phi_{\max } / m v_{\text {th }}^{2}>1$. (There are also asymmetric magnetic mirroring effects, as particles traverse the barrier; however, since in the absence of the effects that we discuss here the magnetic effects, i.e., the $\mu \nabla B_{0}$ forces, being symmetrical, must cancel, we ignore them for the purposes of this calculation. In principle, they would be included in the full transmission function $\boldsymbol{T}$.) Then, the barrier is strong enough to reflect most of the particles coming from region III (Fig. 2) adiabatically with $\Delta \mathcal{E} \approx 0$. In this case, the particles heated are those that travel backwards (from region I), and pass through the resonance region. It can be shown that the transverse heating for these passing particles is approximately $\Delta \mathcal{E}_{\perp}=(\pi / 2) \sqrt{\Lambda} \Phi_{\max }$, where $\sqrt{\Lambda}=\sqrt{L_{B} \omega / v_{z}}$ is the number of cyclotron orbits completed in traversing the resonant region. For transmitted particles, for which, at $\Lambda \gg 1$, the primary effect is heating rather than acceleration, substitute $\chi\left(\boldsymbol{v}_{+}\right)=v_{z c}\left(v_{z c}^{2}+v_{\perp c}^{2}+2 \Delta \mathcal{E}_{+} / m\right)^{3 / 2} v_{\mathrm{th}} \nu_{0}^{-1}$ and $\Delta \mathcal{E}_{+}=(\pi / 2) \kappa \sqrt{\Lambda} m v_{\text {th }}^{2}$ into Eq. (6). For reflected particles, substitute $\chi\left(\boldsymbol{v}_{-}\right)=v_{z c}\left(v_{z c}^{2}+v_{\perp c}^{2}\right)^{3 / 2} v_{\text {th }} \nu_{0}^{-1}$ and $\Delta \mathcal{E}_{-}=0$. Then, the efficiency of the ponderomotive (PM) current drive can be written as

$\frac{\eta_{\mathrm{PM}}}{\eta_{0}}=\frac{2 u}{\pi \kappa \sqrt{\Lambda}}\left[\left(v_{z c}^{2}+v_{\perp c}^{2}+2 \alpha \kappa \sqrt{\Lambda}\right)^{\frac{3}{2}}+\left(v_{z c}^{2}+v_{\perp c}^{2}\right)^{\frac{3}{2}}\right]$,

where $\eta_{0}=e / 2 \pi m R \nu_{0} v_{\text {th }}$ and $v_{z c}$ and $v_{\perp c}$ are measured in units $v_{\text {th }}$.

To gain an appreciation for the approximate efficiency, we can compare the efficiency in this very approximate model of the ponderomotive effect to that achieved in the electroncyclotron current drive (ECCD) scheme [2]. In ECCD, the rf field heats in the perpendicular direction only those particles which travel in one direction. Thus, take $\chi\left(\boldsymbol{v}_{+}\right)=$ $v_{z c}\left(v_{z c}^{2}+v_{\perp c}^{2}+2 \Delta \mathcal{E} / m\right)^{3 / 2} v_{\mathrm{th}} \nu_{0}^{-1}, \Delta \mathcal{E}_{+} \equiv \Delta \mathcal{E}$ for the particles incident on the barrier with positive $v_{z}$, and $\chi\left(\boldsymbol{v}_{-}\right)=$ $-v_{z c}\left(v_{z c}^{2}+v_{\perp c}^{2}\right)^{3 / 2} v_{\text {th }} \nu_{0}^{-1}, \Delta \mathcal{E}_{-}=0$ for those incident on the barrier with negative $v_{z}$. According to Eq. (6), in dimensionless form, the EC current drive efficiency is then given by $\eta_{\mathrm{EC}} / \eta_{0}=3 v_{z c} \sqrt{v_{z c}^{2}+v_{\perp c}^{2}}$ in the limit $\Delta \mathcal{E} \rightarrow 0$. To estimate the ratio $\eta_{\mathrm{PM}} / \eta_{\mathrm{EC}}$, take $\kappa=2, \Lambda=4$; for the normalized energy of 2-D transverse motion, take $v_{\perp c}^{2}=2$; since in wide range of the parameter the ratio of the two efficiencies appears to be insensitive to $v_{z c} \sim 1$, the precise value of the latter is not important and can be taken, say, equal to unity. With these parameters, one gets $\eta_{\mathrm{PM}} / \eta_{\mathrm{EC}} \gtrsim 2$.

What this crude calculation tells us is that the ponderomo- 
tive current drive efficiency can be large, maybe even larger than the EC current drive efficiency. What is necessary, of course, is a much more precise estimate than that provided here including an optimization over the relevant parameters. In particular, if magnetic reflection can be used to lower the ponderomotive barrier necessary to reflect electrons, then the associated heating might, in principle, be made much lower.

The effects calculated here apply equally to ions as well as to electrons. Driving a minority species ion current in a plasma with two ion species can similarly lead to efficient current drive $[5,32]$, and the ions driven by the ponderomotive effects suggested here will lead to similar current drive efficiencies. To drive an ion current, consider two species of ions, one with charge state $Z_{\alpha}$ times the other charge state. Suppose the ions drift relative to each other. Note that in the frame of reference in which the ion current vanishes, the electrons will tend to follow the ions with the higher charge state, since they collide more often with the higher charge ions, resulting in net current, density $J=e n_{\alpha} v_{\alpha} Z_{\alpha}\left(1-Z_{\alpha}\right)$. Since current in a neutral plasma is frame-invariant, the current appears in the lab frame as well. To induce a drift of momentum $p_{\alpha}$, by pushing ions from velocity space location (1) to velocity space location (2), requires power $P_{D}$, such that

$$
\frac{p_{\alpha}}{P_{D}}=m_{\alpha} \frac{\left(v_{z} / \nu\right)_{2}-\left(v_{z} / \nu\right)_{1}}{\Delta \mathcal{E}}
$$

where $\nu=\nu_{e}+\nu_{i}$ is the sum of the collisional slowing down frequencies on electrons and majority ions respectively. Now in the case of minority species current drive, which has recently enjoyed experimental verification [33], if minority ions are, say, cyclotron heated in the perpendicular direction, then velocity space location (2) is nearby location (1), and we get $p_{\alpha} / P_{D}=(3 / 2) m_{\alpha}\left(v_{z} / \nu\right)\left(\nu_{i} / \nu\right)$, where the effect is maximized when $\nu_{e} \approx \nu_{i}$. For the case of ponderomotive barrier reflection, we get an induced drift $p_{\alpha} / P_{D}=$ $2 m_{\alpha}\left(v_{z} / \nu\right) / \Delta \mathcal{E}_{\perp}$, where we used a model two-delta function distribution, like in the case of Eq. (5), except that we assumed that the main current drive effect came from reflection, rather than heating. The main inefficiency occurs from the perpendicular heating, which is related to the maximum ponderomotive potential, as for electrons, by $\Delta \mathcal{E}_{\perp}=(\pi / 2) \sqrt{\Lambda} \Phi_{\max }$. Comparing these two efficiencies, we can see that if the ponderomotive potential is several times the minority ion temperature so as to reflect nearly all impinging minority ions, and if $\Lambda$ is kept small, say, then the current drive efficiency is of the order or can exceed the minority species current drive efficiency.

In summary, these calculations are quite crude, but they illustrate that the ponderomotive current drive effect with sign reversal has an efficiency that can be on the order of the efficiencies of leading resonant $\mathrm{rf}$ current drive mechanisms. Since, however, the new mechanism depends on very different physics, with very different parametric dependencies, there remains the possibility that an optimized implementation of this effect could result in rf current drive efficiencies otherwise unachievable. An important caveat to this speculation is that it remains to identify suitable plasma waves that may be excited in confinement devices of interest and that can be made to achieve the ponderomotive potentials that result in high efficiencies of current generation. A toroidal device of interest for the practice of this effect might be a spherical torus or a stellerator, where the magnetic field strength tends to change greatly along a magnetic field line.

The work is supported by DOE contract DE-AC0276CHO3073. One of us (NJF) is in debt to the late Dr. Christof Litwin for stimulating discussions over many years, in which Dr. Litwin predicted that ponderomotive effects could somehow be put to important use in driving toroidal current.

[1] N. J. Fisch, Phys. Rev. Lett. 41, 873 (1978).

[2] N. J. Fisch and A. H. Boozer, Phys. Rev. Lett. 45, 720 (1980).

[3] N. J. Fisch, Rev. Mod. Phys. 59, 175 (1987).

[4] N. J. Fisch and C. F. F. Karney, Phys. Fluids 24, 27 (1981).

[5] N. J. Fisch, Nuclear Fusion 21, 15 (1981).

[6] N. J. Fisch and J-M. Rax, Phys. Rev. Lett. 69, 612 (1992).

[7] H. Motz and C. J. Watson, in Advances in Electronics and Electron Physics Vol. 23, ed. L. Marton, Academic Press, N. Y. pp. 154-302 (1967).

[8] R. M. O. Galvao, A. G. Elfimov, G. Amarante-Segundo, V. S. Tsypin, L. F. Ruchko, I. C. Nascimento and M. Tendler, Plasma Phys. Control. Fusion 41, A487 (1999).

[9] A. G. Elfimov, Sov. J. Plasma Phys. 17, 223 (1991).

[10] A. G. Elfimov, J. A. Tataronis, and N. Hershkowitz, Phys. Plasmas 1, 2637 (1994).

[11] A. G. Elfimov Phys. Plasmas 8, 2050 (2001).

[12] C. Litwin, Phys. Plasmas 1, 515 (1994).

[13] V. V. Gaponov and M. A. Miller, Sov. Phys. JETP-USSR 7, 168 (1958); V. V. Gaponov and M. A. Miller, Sov. Phys. JETPUSSR 7, 515 (1958).

[14] J. R. Cary and A. N. Kaufman, Phys. Fluids 24, 1238 (1981).

[15] M. Porkolab and M. V. Goldman, Phys. Fluids 19, 872 (1976).

[16] R. Klima, Czech J. Physics B 18, 168 (1968).

[17] J. M. Alimi, P. Mora, and R. Pellat, J. Physique 48, 585 (1987).

[18] E. S. Weibel, Phys. Rev. Lett. 44, 377 (1980).

[19] A. G. Elfimov, R. M. O. Galvao, and I. C. Nascimento, Plasma Sources Science and Technology 7, 410 (1998).

[20] C. Grebogi and H. S. Uhm, Phys. Fluids 31, 1277 (1988).

[21] B. M. Lamb, G. Dimonte, and G. J. Morales, Phys. Fluids 27, 1401 (1984); G. Dimonte, B. M. Lamb, and G. J. Morales, Plas. Phys. 25, 713 (1983).

[22] C. Grebogi, A. N. Kaufman, and R. G. Littlejohn, Phys. Rev. Lett. 43, 1668 (1979).

[23] J. R. Ferron, N. Hershkowitz, R. A. Breun, S. N. Golovato, and R. Goulding, Phys. Rev. Lett. 51, 1955 (1983); Y. Yasaka and R. Itatani, Phys. Rev. Lett. 56, 2811 (1986).

[24] A. J. Lichtenberg and H. L. Berk, Nuclear Fusion 15, 999 (1975).

[25] T. Consoli and R. B. Hall, Nuclear fusion 3, 237 (1963).

[26] T. Hatori and T. Watanabe, Nuclear Fusion 15, 143 (1975).

[27] G. Shvets, N. J. Fisch, and J.-M. Rax, Phys. Rev. Lett. 80, 2598 (1998).

[28] J. J. Martinell, Rev. Mex. Fis. 48, 239 (2002).

[29] A. Kildal, Contrib. Plasma Phys. 39, 349 (1999).

[30] S. Cuperman, C. Bruma, and K. Komoshvili, Phys. Rev. A 311, 221 (2003). 
[31] V. S. Tsypin, A. G. Elfimov, C. A. Deazevedo, and A. S. Deassis, Phys. Plasmas 2, 2784 (1995).

[32] J. Carlsson, T. Hellsten, and J. Hedin, Phys. Plasmas 5, 2885 (1998).
[33] E. Westerhof et al, Nuclear Fusion 42, 1324 (2002); M. J. Mantsinen et al, Plasma Phys. Cont. Nucl. Fusion 44, 1521 (2002). 


\section{External Distribution}

Plasma Research Laboratory, Australian National University, Australia

Professor I.R. Jones, Flinders University, Australia

Professor João Canalle, Instituto de Fisica DEQ/IF - UERJ, Brazil

Mr. Gerson O. Ludwig, Instituto Nacional de Pesquisas, Brazil

Dr. P.H. Sakanaka, Instituto Fisica, Brazil

The Librarian, Culham Laboratory, England

Mrs. S.A. Hutchinson, JET Library, England

Professor M.N. Bussac, Ecole Polytechnique, France

Librarian, Max-Planck-Institut für Plasmaphysik, Germany

Jolan Moldvai, Reports Library, Hungarian Academy of Sciences, Central Research Institute for Physics, Hungary

Dr. P. Kaw, Institute for Plasma Research, India

Ms. P.J. Pathak, Librarian, Institute for Plasma Research, India

Ms. Clelia De Palo, Associazione EURATOM-ENEA, Italy

Dr. G. Grosso, Instituto di Fisica del Plasma, Italy

Librarian, Naka Fusion Research Establishment, JAERI, Japan

Library, Laboratory for Complex Energy Processes, Institute for Advanced Study, Kyoto University, Japan

Research Information Center, National Institute for Fusion Science, Japan

Dr. O. Mitarai, Kyushu Tokai University, Japan

Dr. Jiangang Li, Institute of Plasma Physics, Chinese Academy of Sciences, People's Republic of China

Professor Yuping Huo, School of Physical Science and Technology, People's Republic of China

Library, Academia Sinica, Institute of Plasma Physics, People's Republic of China

Librarian, Institute of Physics, Chinese Academy of Sciences, People's Republic of China

Dr. S. Mirnov, TRINITI, Troitsk, Russian Federation, Russia

Dr. V.S. Strelkov, Kurchatov Institute, Russian Federation, Russia

Professor Peter Lukac, Katedra Fyziky Plazmy MFF UK, Mlynska dolina F-2, Komenskeho Univerzita, SK-842 15 Bratislava, Slovakia

Dr. G.S. Lee, Korea Basic Science Institute, South Korea

Institute for Plasma Research, University of Maryland, USA

Librarian, Fusion Energy Division, Oak Ridge National Laboratory, USA

Librarian, Institute of Fusion Studies, University of Texas, USA

Librarian, Magnetic Fusion Program, Lawrence Livermore National Laboratory, USA

Library, General Atomics, USA

Plasma Physics Group, Fusion Energy Research Program, University of California at San Diego, USA

Plasma Physics Library, Columbia University, USA

Alkesh Punjabi, Center for Fusion Research and Training, Hampton University, USA

Dr. W.M. Stacey, Fusion Research Center, Georgia Institute of Technology, USA

Dr. John Willis, U.S. Department of Energy, Office of Fusion Energy Sciences, USA

Mr. Paul H. Wright, Indianapolis, Indiana, USA 
The Princeton Plasma Physics Laboratory is operated by Princeton University under contract with the U.S. Department of Energy.

\author{
Information Services \\ Princeton Plasma Physics Laboratory \\ P.O. Box 451 \\ Princeton, NJ 08543
}

Phone: 609-243-2750

Fax: 609-243-2751

e-mail: pppl_info@pppl.gov

Internet Address: http://www.pppl.gov 